\title{
Adaptive Fuzzy Decentralized Control of Robot Manipulators
}

\author{
Nasser Sadati and Ehsan Elhamifar \\ Electrical Engineering Department, Sharif University of Technology, Tehran, Iran \\ sadati@sina.sharif.edu ,elhamifar@ee.sharif.edu
}

\begin{abstract}
In this paper an adaptive fuzzy decentralized control algorithm for trajectory tracking of robot manipulators is developed. The proposed decentralized control algorithm allows the overall closed-loop system to be stabilized while making the tracking error to be uniformly ultimately bounded (UUB), without having any prior knowledge of the robot manipulator dynamics. The interconnections in the dynamic equations of each subsystem are considered with unknown nonlinear bounds. The adaptive fuzzy neural networks (AFNNs) are proposed to model the unknown nonlinear dynamics of the robots and the interconnection terms. Using Lyapunov method, the stability of the overall system is investigated.
\end{abstract}

\section{INTRODUCTION}

The control of robot manipulators is challenging due to the inherent high nonlinearity in their dynamics. In classical control theory, it is assumed that the control actions are undertaken by a single controller that has all the available information about the system. Although significant achievements to improve the tracking performance of robots by using centralized schemes have been gained, [1]-[2], but when the degree of freedom (D.O.F) of robots is large they encounter time consuming computations and complexity of the controller structure. Therefore we seek for control methods that reduce these problems by changing the problem to control smaller scale systems by using the decentralized controllers that have the advantage of computation simplicity and low-cost hardware setup. Therefore improving the performance of the tracking problem of robots through decentralized control is an interesting topic.

Earlier literature on the decentralized control methods were focused on control of large-scale linear systems. These works consider subsystems which are linear in a set of unknown parameters [3]-[7]. The others were focused on systems with first order interconnections [3]-[10]. But their results can not guarantee stability when interconnections are of higher order as depicted in [11]. These methods are not applicable to the control of robot manipulators which have highly nonlinear interconnection terms, so we seek for efficient methods that consider the interactions as unknown nonlinear functions and design suitable decentralized controllers to eliminate the effects of these terms in dynamic equations of each subsystem.

In this paper, robust fuzzy decentralized control laws are proposed for the tracking problem of robot manipulators.
The AFNNs are used here as a tool to model the unknown nonlinear dynamics of each mechanical subsystem and eliminate the effects of interaction terms on each subsystem resulted from decomposing the overall robotic system into multiple smaller scales mechanical systems. The main advantage of the proposed approach is that, the interconnections could be considered with arbitrary unknown nonlinear bounds.

The structure of this paper is as follows. In Section II we formulate the dynamics of the overall robotic system and the smaller scale subsystems. Also we define the bounds on interconnections. In Section III we propose a brief review of the AFNNs that we have used them in the paper, then in Section IV we introduce a control input including the outputs of two AFNNs, one for estimating the unknown local dynamics of each subsystem and the other for the unknown nonlinear interactions. In Section $\mathrm{V}$ of the paper the effectiveness of our method is shown by an application example. The paper concludes in Section VI.

\section{PROBLEM STATEMENT}

Consider a robotic system with degree of $n$ described by the following equation

$$
M(q) \ddot{q}+C(q, \dot{q}) \dot{q}+G(q)+d(q, \dot{q}, t)=\tau
$$

where $q$ is the generalized coordinate of the system, $M($.$) is$ the inertia matrix, $C($.$) is related to the coriolis/centrifugal$ torques, $G($.$) is the gravity vector of the system,$ $d($.) denotes the disturbance vector and $\tau$ is the generalized force applied to the system.

Now, according to difficulties of a centralized control algorithm, a large-scale robotic system can be decomposed into $N$ subsystems, each having degree of $n_{i}$ such that

$$
\begin{aligned}
& \sum_{i=1}^{N} n_{i}=n \text {. So we can write (1) as } \\
& M_{i}\left(q_{i}\right) \ddot{q}_{i}+C_{i}\left(q_{i}, \dot{q}_{i}\right) \dot{q}_{i}+G_{i}\left(q_{i}\right)+d_{i}\left(q_{i}, \dot{q}_{i}\right)+\Delta_{i}(q, \dot{q}, t)=\tau_{i}
\end{aligned}
$$

here $d_{i}\left(q_{i}, \dot{q}_{i}\right)$ represents the disturbance term of subsystem $i$ and $\Delta_{i}\left(q_{i}, q_{i}, t\right)$ is the interaction term of other subsystems on the $i$ th one. We can consider one joint or multiple joints as a subsystem to be controlled by a decentralized controller.

Assumption 1: The unknown interconnection vectors satisfy the following inequality 


$$
\left\|\Delta_{i}\left(q_{i}, \dot{q}_{i}, t\right)\right\| \leq \xi_{i 0}+\sum_{j=1}^{N} \xi_{i j}\left(\left\|s_{i}\right\|\right) \quad ; \forall t \in[0, \infty)
$$

where $\xi_{i 0}$ 's are unknown positive constants and $\xi_{i j}($.$) 's are$ unknown smooth functions with $\xi_{i j}(0)=0$. Now by assumption that all the joints are revolute the following properties can be resulted [13].

Property 1: The inertia matrix $M_{i}\left(q_{i}\right)$ is symmetric and positive definite, satisfying the following relation

$$
\underline{\lambda}_{i}\|x\|^{2} \leq x^{T} M_{i} x \leq \bar{\lambda}_{i}\|x\|^{2}
$$

where $\underline{\lambda}_{i}$ and $\bar{\lambda}_{i}$ denote the maximum and minimum eigenvalues of $M_{i}$, respectively. For other matrices $\bar{\lambda}($.) and $\underline{\lambda}$ (.) denote the maximum and minimum eigenvalues of (.), respectively. Also the Euclidean norm is described by $\|x\|^{2}=x^{T} x$.

Property 2: $\dot{M}_{i}\left(q_{i}\right)-2 C_{i}\left(q_{i}, \dot{q}_{i}\right)$ is skew-symmetric.

\section{AdAPTIVE FuZZY NEURAL NETWORKS}

The basic configuration of a fuzzy logic system consists of a fuzzifier, some fuzzy IF-THEN rules, a fuzzy inference engine and a defuzzifier. The fuzzy inference engine uses the fuzzy IF-THEN rules to perform a mapping from an input vector $x=\left(x_{1}, \quad x_{2}, \ldots, x_{r}\right)^{T}$ to an output $y$. The $i$ th fuzzy rule is written as

$$
R^{i} \text { : If } x_{1} \text { is } A_{1}^{i} \text { and } \ldots \text { and } x_{r} \text { is } A_{r}^{i} \text {, then } y \text { is } y^{i}
$$

where $A_{1}^{i}, A_{2}^{i}, \ldots, A_{r}^{i}$ are fuzzy variables and $y^{i}$ is the center of the $i$ th output membership function. By using singleton fuzzifier, product inference and center-average defuzzifier, the output of the fuzzy system can be expressed as

$$
F=\frac{\sum_{i=1}^{p} b_{i} \xi_{i}(x)}{\sum_{i=1}^{p} \xi_{i}(x)}=B^{T} \xi(x)
$$

where $\mu_{A_{j}^{i}}\left(x_{j}\right)$ is the membership function value of the fuzzy variable $x_{j}, \quad p$ is the number of fuzzy rules, $B=\left[\begin{array}{llll}y^{1} & y^{2} & \ldots & y^{p}\end{array}\right]^{T}$ is the adjustable parameter vector ( composed of consequent parameters ), and

$$
\begin{gathered}
\xi^{T}(x)=\left[\begin{array}{cccc}
\xi_{1}(x) & \xi_{2}(x) & \ldots & \xi_{p}(x)
\end{array}\right] / \sum_{i=1}^{r} \xi_{i}(x) \\
\xi_{i}(x)=\prod_{j=1}^{r} \mu_{A_{j}^{i}}\left(x_{j}\right)
\end{gathered}
$$

is the fuzzy basis function (FBF).

Given a fuzzy neural network, it is possible to approximate a wide variety of functions simply by making different choices for $B$. In particular if there is a sufficient number of rules within the network, then there exist some $B^{*}$ such that

$$
\sup \left\|B^{* T} \xi(x)-f(x)\right\| \leq \rho_{M}, \forall x \in S_{x}
$$

where $S_{X}$ is a compact set and $\rho_{M}>0$ is a finite constant provided $f(x)$ is continuous [12]. So we can express $f(x)=B^{T} \xi(x)+\rho(x)$ with $\|\rho(x)\| \leq \rho_{M}$ when $x \in S_{x}$ Note that even though AFNNs are linear in a set of adjustable parameters, a function which is not linear in an independent set of parameters could be approximated. It should be noted that any universal approximator which is linear in adjustable parameters may be considered such as RBF neural networks, Takagi-Sugeno fuzzy systems, CMAC networks, etc,.

\section{CONTROLLER DESIGN}

Assuming the desired trajectory for the $i$ th subsystem to be twice differentiable, define the following relations

$$
\begin{gathered}
\tilde{q}_{i}=q_{i}-q_{d i} \\
\dot{q}_{r i}=\dot{q}_{d i}-\Lambda_{i} \tilde{q}_{i} \\
s_{i}=\dot{q}_{i}-\dot{q}_{r i}=\dot{\tilde{q}}_{i}+\Lambda_{i} \tilde{q}_{i}
\end{gathered}
$$

where $\tilde{q}_{i}$ is the tracking error, $\dot{q}_{r i}$ is the reference velocity and $s_{i}$ is the residual error for the $i$ th mechanical subsystem. Now substituting (4) into the dynamic equation of the subsystem $i$, described by (2), results in

$$
\begin{aligned}
& M_{i}\left(q_{i}\right) \dot{s}_{i}=-C_{i}\left(q_{i}, \dot{q}_{i}\right) s_{i}-\Delta_{i}\left(q_{i}, \dot{q}_{i}, t\right)+\tau_{i} \\
& \quad-\left(M_{i}\left(q_{i}\right) \ddot{q}_{r i}+C_{i}\left(q_{i}, \dot{q}_{i}\right) \dot{q}_{r i}+G_{i}\left(q_{i}\right)+d_{i}\left(q_{i}, \dot{q}_{i}\right)\right)
\end{aligned}
$$

Here we assume the dynamics of the robot manipulator are unknown, so according to the general approximation property of the AFNNs we can write

$$
\begin{array}{r}
M_{i}\left(q_{i}\right) \ddot{q}_{r i}+C_{i}\left(q_{i}, \dot{q}_{i}\right) \dot{q}_{r i}+G_{i}\left(q_{i}\right)+d_{i}\left(q_{i}, \dot{q}_{i}\right) \\
=W_{i}^{* T} \Phi_{i}\left(q_{i}, \dot{q}_{i}, \dot{q}_{r i}, \ddot{q}_{r i}\right)+w_{i}
\end{array}
$$

where $w_{i}$ 's are the minimum approximation errors of the fuzzy neural networks which can be made sufficiently small by choosing the appropriate fuzzy basis functions $\Phi_{i}($.$) .$ Also assume that $\left\|w_{i}\right\| \leq w_{M i}$.

Now, consider the control law to be given as follows

$$
\begin{gathered}
\tau_{i}=-K_{i} s_{i}+W_{i}^{T} \Phi_{i}(.)-r_{i} \\
r_{i}=Z_{i}{ }^{T} \eta_{i}\left(\left\|s_{i}\right\|\right) s_{i}+\theta_{i} s_{i}
\end{gathered}
$$

where the design matrices $K_{i}$ 's are positive definite and the constants $\theta_{i}>0$ are chosen to be sufficiently large. $Z_{i}^{T} \eta_{i}\left(\left\|s_{i}\right\|\right)$ are the outputs of the second AFNNs with basis functions $\eta_{i}\left(\right.$.) and inputs $\left\|s_{i}\right\|$. The effect of this term and the related details would be discussed in the proof of the following theorem. 
Note that the control law (7) is apparently in a decentralized manner and its performance can be summarized using the following theorem.

Theorem: Consider the $n$ degree of freedom robotic system described by (1) and decompose it into $N$ subsystems with dynamics given by (2). Now choosing the control input for the $i$ th subsystem given by (7), the solution $s_{i}(t)$ is uniformly ultimately bounded. Moreover the ultimate bound can be made arbitrary small choosing appropriate gains. For a desired bound $\beta_{i}$, there exist controller gains and suitable update laws for the AFNNs such that for a finite time $t_{r i}$ :

If $\left\|s_{i}(0)\right\| \leq a_{i}$ then $\left\|s_{i}\right\| \leq \beta_{i} \sqrt{\frac{\bar{\lambda}_{i}}{\underline{\lambda}_{i}}} \quad \forall t \geq t_{r i}$

Proof: Consider the Lyapunov function of the system to be given by

$$
V=\sum_{i=1}^{N} V_{i}=\sum_{i=1}^{N} V_{i 1}+V_{i 2}
$$

where

$$
\begin{aligned}
& V_{i 1}=\frac{1}{2} s_{i}{ }^{T} M_{i} s_{i} \\
& V_{i 2}=\frac{1}{2} \operatorname{tarce}\left(\tilde{W}_{i}^{T} \Gamma_{i}{ }^{-1} \tilde{W}_{i}\right)+\frac{1}{2} \gamma_{i}{ }^{-1} \tilde{Z}_{i}{ }^{T} \tilde{Z}_{i}
\end{aligned}
$$

also $\Gamma_{i}$ 's and $\gamma_{i}{ }^{\prime} s$ are positive definite matrices and positive constants, respectively. Define $\tilde{W}_{i}=W_{i}-W_{i}^{*}$ and similarly $\tilde{Z}_{i}=Z_{i}-Z_{i}^{*}$.

Now taking the first derivative of $V_{i}$, by using (6) and (7) and assumption 1, we obtain

$$
\begin{aligned}
\sum_{i=1}^{N} \dot{V}_{i 1} \leq & \sum_{i=1}^{N}\left(-s_{i}^{T} K_{i} s_{i}+s_{i}^{T} \tilde{W}_{i}^{T} \Phi_{i}(.)+\left\|s_{i}\right\|\left(\xi_{i 0}+w_{M i}\right)+s_{i}^{T} r_{i}\right) \\
& +\sum_{i=1}^{N}\left(\left\|s_{i}\right\| \sum_{j=1}^{N} \xi_{i j}\left(\left\|s_{i}\right\|\right)\right)
\end{aligned}
$$

Considering the last term of the right hand side of the above inequality we get

$$
\begin{gathered}
\sum_{i=1}^{N}\left(\left\|s_{i}\right\| \sum_{j=1}^{N} \xi_{i j}\left(\left\|s_{j}\right\|\right)\right)=\sum_{i=1}^{N} \sum_{j=1}^{N}\left\|s_{i}\right\|\left\|s_{j}\right\| \varepsilon_{i j}\left(\left\|s_{j}\right\|\right) \\
\leq \sum_{i=1}^{N} \sum_{j=1}^{N}\left(\frac{1}{2}\left\|s_{i}\right\|^{2}+\frac{1}{2}\left\|s_{j}\right\|^{2} \varepsilon_{i j}{ }^{2}\left(\left\|s_{j}\right\|\right)\right) \\
=\sum_{i=1}^{N}\left\|s_{i}\right\|^{2}\left(\frac{N}{2}+\sum_{j=1}^{N} \varepsilon_{i j}^{2}\left(\left\|s_{i}\right\|\right)\right)
\end{gathered}
$$

Here we have used the property that for the smooth function $\xi_{i j}\left(\left\|s_{j}\right\|\right)$ there exists another smooth function $\varepsilon_{i j}\left(\left\|s_{j}\right\|\right)$ such that $\xi_{i j}\left(\left\|s_{j}\right\|\right)=\left\|s_{j}\right\| \varepsilon_{i j}\left(\left\|s_{j}\right\|\right)$. Also in the above inequality the fact that $2 a b \leq a^{2}+b^{2}$ has been used.
We can estimate the unknown nonlinear function $\frac{N}{2}+\sum_{j=1}^{N} \varepsilon_{i j}^{2}\left(\left\|s_{i}\right\|\right)$ by a second AFNN in the form of

$$
\frac{N}{2}+\sum_{j=1}^{N} \varepsilon_{i j}^{2}\left(\left\|s_{i}\right\|\right)=Z_{i}^{* T} \eta_{i}\left(\left\|s_{i}\right\|\right)+z_{i}
$$

where $z_{i}$ 's are the minimum approximation errors of this AFNN with $\left|z_{i}\right| \leq z_{M i}$. Now substituting $r_{i}$ from (7) and using (9) we obtain

$$
\begin{aligned}
\sum_{i=1}^{N} \dot{V}_{i 1} \leq & \sum_{i=1}^{N}\left(-s_{i}{ }^{T} K_{i} s_{i}+s_{i}{ }^{T} \tilde{W}_{i}^{T} \Phi_{i}(.)-\left\|s_{i}\right\|^{2} \tilde{Z}_{i}^{T} \eta_{i}(.)\right) \\
& +\sum_{i=1}^{N}\left(\left\|s_{i}\right\|\left(\xi_{i 0}+w_{M i}\right)-\left\|s_{i}\right\|^{2}\left(\theta_{i}-z_{M i}\right)\right)
\end{aligned}
$$

Differentiating $V_{i 2}$ and substituting in the overall derivative of $V$ and choosing the update laws for the weights of the AFNNs in the form of

$$
\begin{gathered}
\dot{W}_{i}=\Gamma_{i} \Phi_{i}(.) s_{i}{ }^{T} \\
\dot{Z}_{i}=-\gamma_{i} \eta_{i}(.)\left\|s_{i}\right\|^{2}
\end{gathered}
$$

we get

$$
\dot{V} \leq \sum_{i=1}^{N}-\underline{\lambda}\left(K_{i}\right)\left\|s_{i}\right\|^{2}+\left\|s_{i}\right\|\left(\xi_{i 0}+w_{M i}\right)-\left\|s_{i}\right\|^{2}\left(\theta_{i}-z_{M i}\right)
$$

$Z_{M i}$ is the bound on the minimum approximation error that could be eliminated in the above inequality by choosing sufficient large values for $\theta_{i}$ 's so that $\theta_{i}-z_{M i} \approx \theta_{i}>0$.

Thus for $\left\|s_{i}\right\| \geq \beta_{i}$ we have $\dot{V}_{i} \leq-\underline{\lambda}\left(K_{i}\right)\left\|s_{i}\right\|^{2}<0$, where

$$
\beta_{i}=\frac{\xi_{i 0}+w_{M i}}{\theta_{i}}
$$

Note that $\dot{V}(t)$ in (12) is bounded as a function of $\left\|s_{i}\right\|$, thus in view of the Appendix I, this results that $\left\|s_{i}\right\| \leq s_{M i}$ for all $t$, where $s_{M i}$ is defined in (15) and $\left\|s_{i}\right\|$ will eventually be smaller than or equal to $\beta_{i} \sqrt{\frac{\bar{\lambda}_{i}}{\underline{\lambda}_{i}}}$.

Now we show that $s_{i}$ will satisfy (8) in a finite time. If $\left\|s_{i}(0)\right\| \leq \beta_{i} \leq \beta_{i} \sqrt{\bar{\lambda}_{i} / \underline{\lambda}_{i}}$, then $t_{r i}=0$. The reason is that $\dot{V}$ is negative whenever $\left\|s_{i}(t)\right\| \geq \beta_{i}$ so that the maximum value that $\left\|s_{i}\right\|$ may reach is defined by $\beta_{i} \sqrt{\bar{\lambda}_{i} / \underline{\lambda}_{i}}$, thus $\left\|s_{i}(t)\right\| \leq \beta_{i} \sqrt{\bar{\lambda}_{i} / \underline{\lambda}_{i}}, \forall t$. 
If $\beta_{i} \leq\left\|s_{i}(0)\right\| \leq \alpha_{i}$, then one has from (12) and property 1 that $\left\|s_{i}\right\| \leq\left\|s_{i}(0)\right\| \exp \left(-\frac{\lambda\left(K_{i}\right)}{2 \bar{\lambda}_{i}} t\right)$, from which a finite value for $t_{r i}$ can be found.

Q.E.D.

Remark 1) It is obvious that by choosing the larger values for $\theta_{i}$, the maximum bound on $\left\|s_{i}\right\|$ would be decreased. Although this improves the tracking performance, it is clear that in return we must apply larger values for control inputs that is not desirable from practical point of view.

\section{Simulation RESUltS}

In order to demonstrate the performance of the proposed adaptive fuzzy decentralized controller, for simplicity we consider a two link planar robot for numerical simulations, as shown in Fig. 1. It is obvious that the proposed method is applicable to any large-scale robotic manipulator. The dynamic matrices and vectors are defined as

$$
M(q)=\left[\begin{array}{ll}
M_{11} & M_{12} \\
M_{21} & M_{22}
\end{array}\right]
$$

with

$$
\begin{aligned}
& M_{11}=\left(m_{1}+m_{2}\right) a_{1}^{2}+m_{2} a_{2}^{2}+2 m_{2} a_{1} a_{2} \cos \left(q_{2}\right) \\
& M_{12}=M_{21}=m_{2} a_{2}^{2}+m_{2} a_{1} a_{2}\left(1+\cos \left(q_{2}\right)\right) \\
& M_{22}=m_{2} a_{2}^{2}
\end{aligned}
$$

and

$$
C(q, \dot{q})=\left[\begin{array}{cc}
-\bar{C} \dot{q}_{2} & -\bar{C}\left(\dot{q}_{1}+\dot{q}_{2}\right) \\
\bar{C} \dot{q}_{1} & 0
\end{array}\right]
$$

with $\bar{C}=m_{2} a_{1} a_{2} \sin \left(q_{2}\right)$. Also

$$
G(q)=\left[\begin{array}{l}
g_{1}(q) \\
g_{2}(q)
\end{array}\right]
$$

where

$$
\begin{aligned}
& g_{1}(q)=\left(m_{1}+m_{2}\right) a_{1} \cos \left(q_{2}\right)+m_{2} a_{2} \cos \left(q_{1}+q_{2}\right) \text { and } \\
& g_{2}(q)=m_{2} a_{2} \cos \left(q_{1}+q_{2}\right) \text { with } q=\left[\begin{array}{ll}
q_{1} & q_{2}
\end{array}\right]^{T} . \text { The }
\end{aligned}
$$

values of the parameters in the above dynamic vectors and matrices are defined by

$a_{1}=1 \mathrm{~m}, a_{2}=0.8 \mathrm{~m}, m_{1}=1 \mathrm{~kg}, m_{2}=0.8 \mathrm{~kg}$.

The simulations have been performed by $\theta_{i}=20$ and $K_{i}=$ diag $(15,15)$ with the initial conditions for the joint variables to be

$$
q_{1}(0)=0, \dot{q}_{1}(0)=0, q_{2}(0)=0.2, \dot{q}_{2}(0)=0 .
$$

Also the desired trajectory for the joints of the robot is given by

$$
\left[\begin{array}{l}
q_{d 1} \\
q_{d 2}
\end{array}\right]=\left[\begin{array}{c}
-0.3 \cos (\pi t) \\
0.3 \sin (\pi t)
\end{array}\right]
$$

By applying the control input (7) to each joint of the planar robot, the joint 1 is shown to follow the desired trajectory with good performance in Fig. 2. The residual error for joint
1 is shown to decrease to a small value near zero in Fig. 3 but it could be decreased by choosing a larger value for $\theta_{i}$. The simulation result in Fig. 4 shows that the joint 2 has a very good performance in transient response of the tracking. In Fig. 5 the residual error for joint 2 is shown to decrease into a small value that can be reduced by increasing the value of $\theta_{i}$. Fig. 6 and Fig. 7 show that the decentralized control inputs for both joints are in a desirable range of variations.

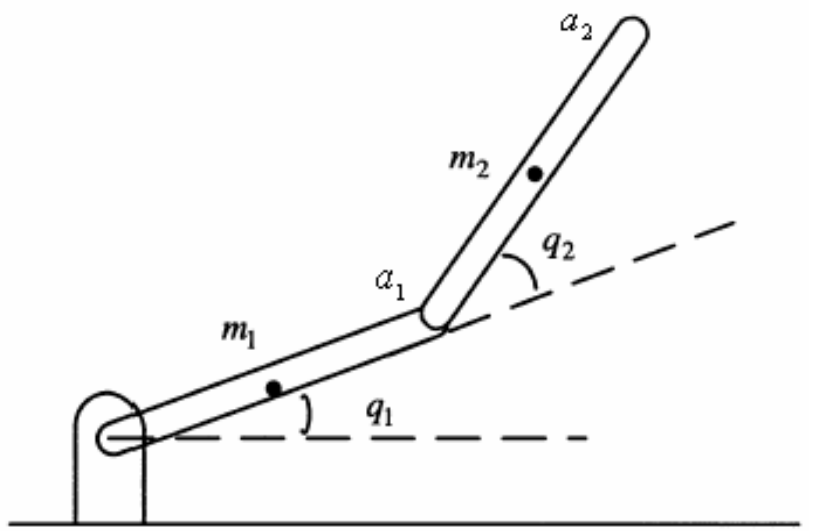

Fig. 1. Two link planar robot.

\section{CONCLUSION}

In this paper a fuzzy neural network decentralized control scheme for robotic manipulators is presented which can be effectively applied to large-scale robot manipulators. It is assumed that the interconnections have unknown nonlinear bounds. It is shown how by using a fuzzy neural network the effects of these interconnections on the performance of each mechanical subsystem have been eliminated. The nonlinear dynamics of each subsystem is also modeled by another fuzzy neural network. The simulation results show that the proposed approach outperforms the decentralized method suggested in [7]. This is mainly because of having a more general structure to model the interconnections.

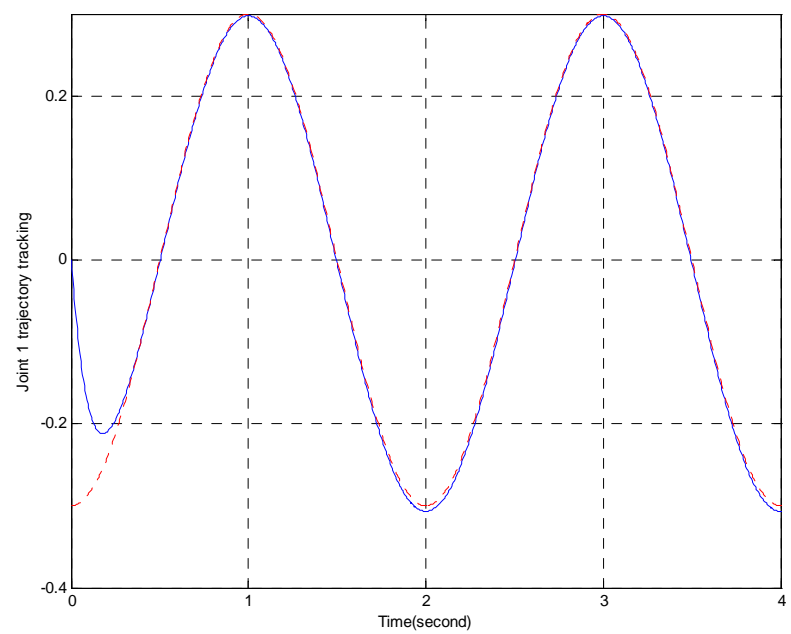

Fig. 2. Trajectory tracking for joint $1 ; q_{1}(-), q_{d 1}(\ldots)$. 


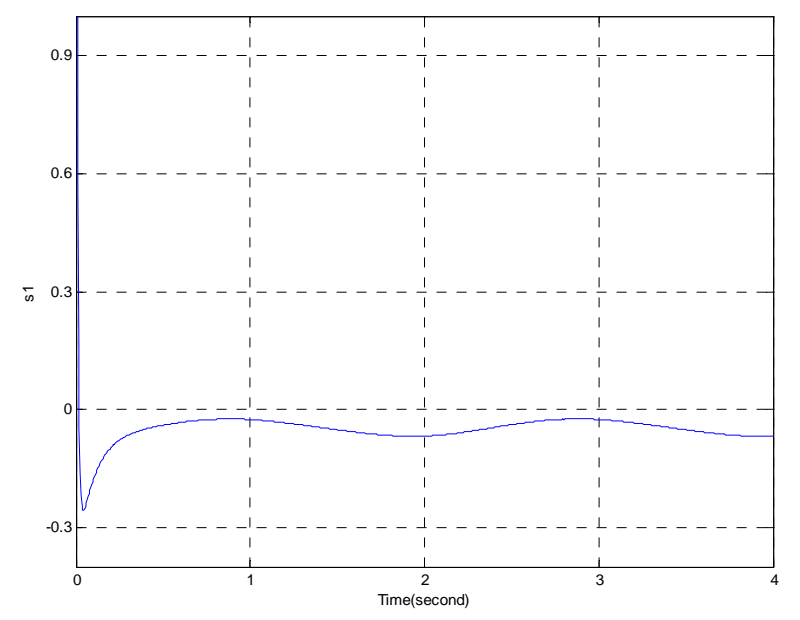

Fig. 3. Residual error for joint 1.

\section{APPENDIX}

For carrying out the mathematical development of the proof of the proposed theorem, consider the following discussion. For the Lyapunov candidate function $V(t)$ given in the proof of the theorem and using property 1, if $\dot{V}(t) \leq 0$ for $t \geq t_{0}$, with $t_{0} \geq 0$, then the norm of $s_{i}$ is bounded by

$$
\left\|s_{i}\right\| \leq \sqrt{\bar{\lambda}_{i} / \underline{\lambda}_{i}}\left\|s_{i}\left(t_{0}\right)\right\|
$$

In other words, as long as $\dot{V}(t)$ is negative or zero, $\left\|s_{i}\right\|$ will be bounded as a function of $\left\|s_{i}\left(t_{0}\right)\right\|$. In particular, if $\left\|s_{i}(0)\right\| \leq a_{i}$, the maximum value of $\left\|s_{i}\right\|$ which may take can be given by

$$
s_{M i}=a_{i} \sqrt{\frac{\bar{\lambda}_{i}}{\underline{\lambda}_{i}}}
$$

However, for some values of $\left\|s_{i}\right\|, \dot{V}(t)$ may become positive. The key point is that even in this case, $\left\|s_{i}\right\|$ must not become larger than $s_{M i}$, and $\dot{V}(t)$ must be bounded for all $t$. Furthermore, $\left\|s_{i}\right\|$ must not be larger than $\beta_{i} \sqrt{\bar{\lambda}_{i} / \underline{\lambda}_{i}}$ for some finite time $t_{r i} \geq 0$. This equivalently means that $s_{i}$ will satisfy (8). So it must be rendered that

$$
\dot{V}(t)<0, \quad \text { if } \beta_{i} \leq\left\|s_{i}\right\| \leq s_{M i}
$$

If $\left\|s_{i}\right\|$ is smaller than $\beta_{i}, \dot{V}(t)$ may become positive, and thus $\left\|s_{i}\right\|$ may increase its value. If $\dot{V}(t)$ is bounded for all $t$, then $\left\|s_{i}\right\|$ cannot have a finite escape time. Thus, since for $\left\|s_{i}\right\| \equiv \beta_{i}, \dot{V}(t)$ is negative, according to (14) the maximum value of $\left\|s_{i}\right\|$ which may take is given by

$$
\left\|s_{i}\right\| \leq \beta_{i} \sqrt{\bar{\lambda}_{i} / \underline{\lambda}_{i}}
$$

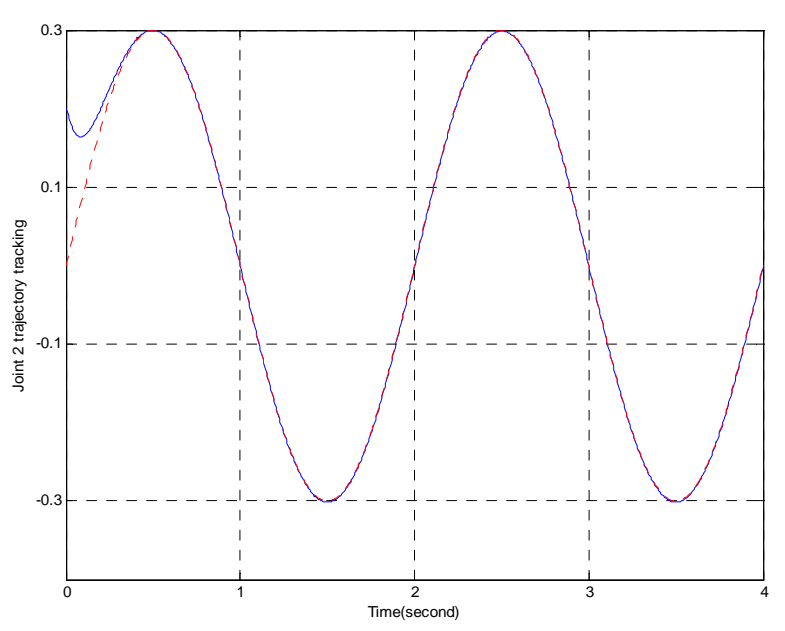

Fig. 4. Trajectory tracking for joint $2 ; q_{2}(-), q_{d 2}(\ldots)$.

Note that one must choose $\beta_{i} \leq s_{M i}$, so that the maximum value of $\left\|s_{i}\right\|$ can be computed from the chosen $a_{i}$ in (8). In conclusion, if (16) holds with $\left\|s_{i}(0)\right\| \leq a_{i}$ and $\dot{V}(t)$ is bounded for all $t$, then the Theorem can be proven. We show in the proof of the theorem that positive gain $\theta_{i}$ can be selected to achieve this goal.

\section{REFERENCES}

[1] J.-J. E. Slotin and W. Li, "On the adaptive control of robot manipulators," Int. J. Robotics Res., vol. 6,no. 3,pp. 49-59, 1987.

[2] M. W. Spong, J. S. Thorp, and J. W. Kleinwaks, "Robust microprocessor control of robot manipulator," Automatica, vol. 23, no. 3, pp. 373-379, 1987.

[3] P. A. Ioannou, "Decentralized adaptive control of interconnected systems," IEEE Trans. Automat. Contr., vol. AC-31, pp. 291-298, 1986.

[4] L. C. Fu, "Robust adaptive decentralized control of robot manipulators," IEEE Trans. Automat. Contr., vol. 37, pp. 106-110, 1992.

[5] S. Sheikholeslam and C. A. Desor, "Indirect adaptive control of a class of interconnected nonlinear dynamical systems," Int. J. Control, vol. 57, no. 3, pp. 743-765, 1993.

[6] C. Wen, "Decentralized adaptive regulation," IEEE Trans. Automat. Contr., vol. 39, pp. 2163-2166, 1994.

[7] Y. Tang, M. Tomizuka, G. Guerrero, and G. Montemayor, "Decentralized robust control of mechanical systems," IEEE Trans. Automat. Contr., vol. 45, no. 4, 2000.

[8] S. N. Huang and H. H. Shao, "Robust stability analysis of uncertain large-scale systems," Control Comput., vol. 23, no. 1, pp. 1-5, 1995.

[9] H. Seraji, "Decentralized adaptive control of manipulators: theory, simulation, and experimentation," IEEE Trans. Robot. Automat. vol. 5, pp. 183-201, 1989.

[10] S. N. Huang, H. H. Shao, and Z. J. Zhang, "Stability analysis of largescale systems with delays," Syst. Control Lett., vol. 25, pp. 75-78, 1995.

[11] L, Shi and S. K. Singh, "Decentralized adaptive controller design for large-scale systems with higher order interconnections," IEEE Trans. Automat. Contr., vol. 37, pp. 1106-1118, 1992.

[12] J. A. Leonard, M. A. Kramer, and L. H. Ungar, "Using radial basis functions to approximate a function and its error bounds," IEEE Trans. Neural Networks, vol. 3, pp. 624-627, 1992.

[13] L. Sciavicco and B. SWsiciliano, Modeling and Control of Robot Manipulators, $2^{\text {nd }}$ ed. London, U. K.: Springer 


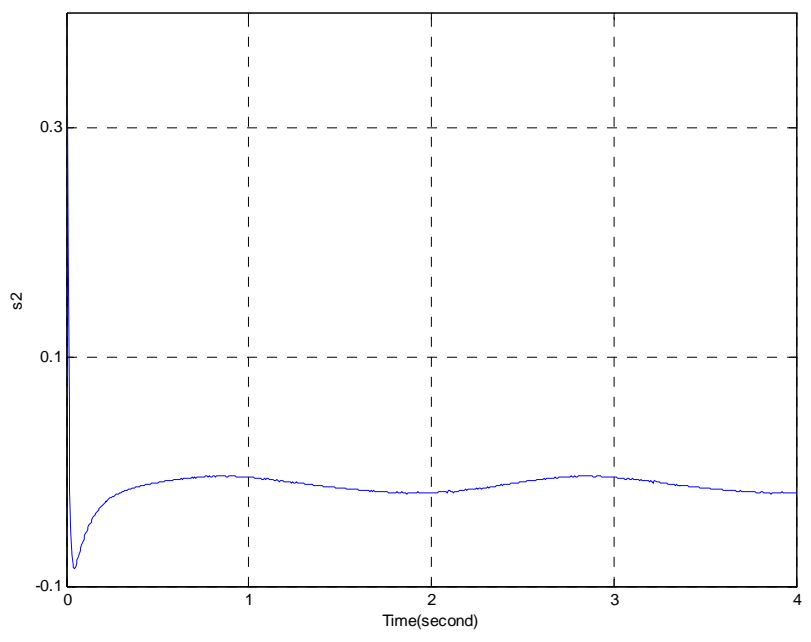

Fig. 5. Residual error for joint 2.

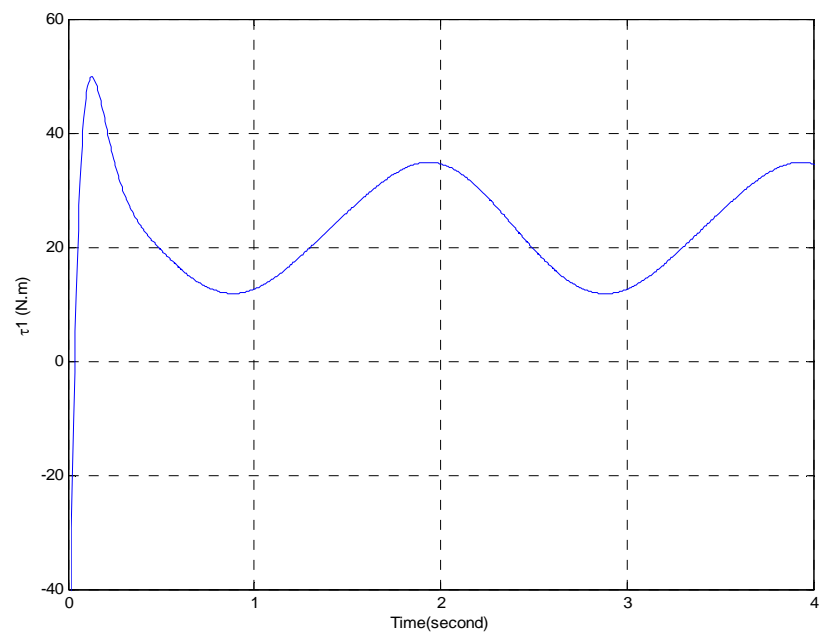

Fig. 6. Joint 1 control input $\left(\tau_{1}\right)$.

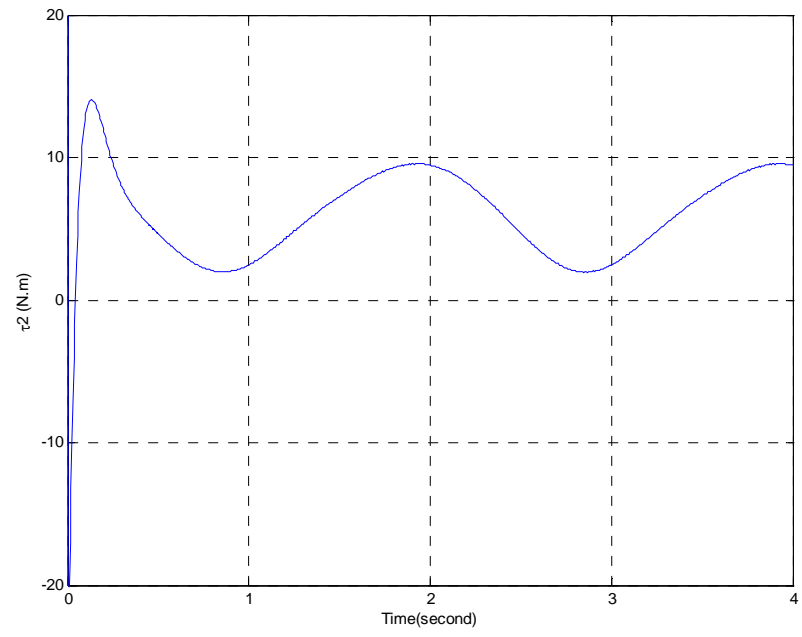

Fig. 7. Joint 2 control input ( $\left.\tau_{2}\right)$. 